\title{
Massa Óssea em Pacientes com Anorexia Nervosa
}

\author{
Bone Mass in Patients with Anorexia Nervosa
}

Evaldo dos Santos ${ }^{1}$, Rosane Pilot Pena Ribeiro ${ }^{3}$, José Ernesto dos Santos ${ }^{2}$ Ana Carolina Japur de Sá Rosa e Silva ${ }^{1}$, Marcos Felipe Silva de Sá ${ }^{1}$

\begin{abstract}
RESUM0
Objetivo: avaliar a ingestão diária de cálcio e estabelecer sua correlação com a densidade óssea de pacientes com anorexia nervos.

Pacientes e Métodos: quatorze mulheres com anorexia nervosa registraram, em diário alimentar padronizado, sua ingestão em 24 horas e foram submetidas ao exame de densitometria óssea. A análise estatística foi feita pelos testes do $\chi^{2}$ e correlação de Pearson, adotando-se como significância estatística $p<0,05$.

Resultados: a média da densidade óssea na coluna lombar foi de 0,95 $\pm 0,15$ e no colo do fêmur foi de 0,88 $\pm 0,26$. Houve correlação significativa entre o tempo de amenorréia e a perda de massa óssea, tanto na coluna lombar $(r=-0,65 ; p=0,01)$ como no fêmur $(r=-0,71$; ( $p=0,0068)$. Com exceção de uma paciente, todas apresentavam ingestão de cálcio inferior ao recomendado pelo $\mathrm{RDA}$, com média de $554,5 \mathrm{mg} /$ dia (variando de 120 a $840 \mathrm{mg} / \mathrm{dia}$ ). Observou-se que 64\% das pacientes apresentavam algum grau de perda de massa óssea (osteopenia ou osteoporose) na coluna e $57 \%$ no fêmur. Das seis pacientes com déficit na ingestão de cálcio inferior a 60\%, nenhuma apresentou osteoporose, ao passo que das oito pacientes com déficit superior a $60 \%$ na ingestão de cálcio, três tinham osteoporose.

Conclusão: Pacientes com anorexia nervosa têm baixa ingestão de cálcio e perda significativa de massa óssea, que estão diretamente relacionadas entre si. A baixa densidade óssea está, também, correlacionada com o tempo de amenorréia.
\end{abstract}

PALAVRAS-CHAVE: Anorexia nervosa, Massa óssea, Osteoporose.

Introdução

A anorexia nervosa (AN) está relacionada a significativa morbidade, estando a amenorréia prolongada presente na maioria dos casos. Por acometer adolescentes, incide em período crítico de formação do esqueleto e, portanto, uma das complicações mais graves é a redução da massa óssea. A incidência de osteopenia pode chegar a 90\% entre estas jovens, sendo que um terço delas tem osteoporose $^{1}$.

${ }^{1}$ Departamento de Ginecologia e Obstetrícia da FMRP-USP. ${ }^{2}$ Departamento de Clínica Médica da FMRP-USP.

${ }^{3}$ Escola de Enfermagem de Ribeirão Preto-USP.

Correspondência:

Marcos Felipe Silva de Sá

Departamento de Ginecologia e Obstetrícia

Faculdade de Medicina de Ribeirão Preto - Universidade de São Paulo

14049-900 - Ribeirão Preto-SP

Fone/Fax: (16) 602-2231

e-mail: mfsdsa@fmrp.usp.br
A redução de massa corporal e o tempo de amenorréia são variáveis importantes, claramente relacionadas à perda de massa óssea. Entretanto, outros fatores têm sido apontados como facilitadores ou desencadeadores do aparecimento da osteopenia. São citados o hipoestrogenismo (relacionado evidentemente à amenorréia), o tempo de evolução da doença, a idade e o grau de desnutrição ${ }^{2,3}$. Neste último caso teria grande importância a baixa ingestão de cálcio e outros nutrientes importantes para a formação da massa óssea. Entretanto, quando se analisam os possíveis fatores causais, no momento do tratamento, a correção do déficit por si só não parece ser suficiente para recuperação da massa óssea. Assim, a simples reposição de cálcio e suplementação multivitamínica nem sempre são suficientes para prevenir a perda de massa óssea ${ }^{4}$, o que coloca em dúvida o papel da baixa ingestão de cálcio como fator preponderante neste processo. 
Desta forma, o objetivo deste trabalho foi avaliar a densidade óssea de pacientes com AN e estabelecer sua correlação com a ingestão diária de cálcio.

\section{Pacientes e Métodos}

Foi realizado estudo clínico transversal, prospectivo, no qual foram incluídas 14 mulheres com AN, que foram atendidas no Ambulatório de Distúrbios de Conduta Alimentar do Hospital das Clínicas da Faculdade de Medicina de Ribeirão Preto-USP, no período de 1995 a 2001 . O diagnóstico de AN seguiu os critérios da American Psychiatric Association $^{5}$, segundo o qual a anorexia nervosa é caracterizada por recusa em manter o peso corporal ideal ou acima do peso mínimo para idade e estatura; medo intenso de ganhar peso ou tornarse obeso, mesmo quando este é inferior ao peso ideal; distúrbios de imagem corporal e amenorréia em mulheres pós-menarca ${ }^{5}$. Foi considerado para avaliação do índice de massa corporal (IMC) o valor normal de referência entre 19 e $24 \mathrm{~kg} / \mathrm{m}^{2}$. A amenorréia foi definida como a ausência da menstruação por três ciclos consecutivos.

A ingestão de cálcio de cada paciente foi obtida pelo registro nas 24 horas, utilizando-se o Programa de Apoio à Nutrição do Hospital das Clínicas da FMRP-USP, por meio de anotação rigorosa da alimentação em um diário alimentar, a partir do qual, utilizando-se tabela de valores nutricionais de cada alimento, era calculada a ingestão diária de cálcio. Para comparação foi utilizada a ingestão diária de cálcio recomendada pelo RDA (Recommended Dietary Allowances) ${ }^{6}$ e verificado o déficit na ingestão de cálcio. No mesmo dia as pacientes foram submetidas ao exame de densitometria óssea (DMO) utilizando-se equipamento de dupla radiação (Dexa). Foram consideradas densidades ósseas normais aquelas com variações até um desvio padrão abaixo da média de uma mulher adulta jovem. Variações entre 1 e 2,5 desvios padrões foram consideradas osteopênicas e maiores que 2,5 desvios padrões, rotuladas como osteoporóticas.

A análise estatística foi feita pelos testes do $\chi^{2}$ para cálculo da diferença entre a ingestão diária de cálcio real e o recomendado pelo $\mathrm{RDA}^{6}$, e correlação de Pearson para avaliar correlação entre a massa óssea e o déficit na ingestão diária de cálcio e a massa óssea e o tempo de amenorréia, adotando-se como significância estatística $\mathrm{p}<0,05$. Este trabalho teve aprovação do Comitê de Ética em Pesquisa do HC-FMRP.

\section{Resultados}

A idade das pacientes variou de 15 a 34 anos, com mediana de 20,5 anos e a mediana do IMC foi de $15,7 \mathrm{~kg} / \mathrm{m}^{2}$, com variação de 11,8 a $20,9 \mathrm{~kg} /$ $\mathrm{m}^{2}$. O tempo de amenorréia foi de 6 a 72 meses, com mediana de 12,5 meses.

Com base nas anotações do registro diário de ingestão alimentar, observamos que a ingestão diária de cálcio teve mediana de $584,0 \mathrm{mg} /$ dia, com variação de 120,0 a $840 \mathrm{mg} /$ dia. A ingestão de cálcio de cada paciente foi comparada aos valores recomendados segundo o RDA (1989) ${ }^{6}$ para o cálcio: $1200 \mathrm{mg} /$ dia para pacientes de 10 a 18 anos de idade e $800 \mathrm{mg} /$ dia para pacientes entre 19 e 50 anos. Observou-se que, à exceção de uma paciente, todas as demais apresentavam ingestão inferior ao recomendado pelo $\mathrm{RDA}^{6}$, com diferenças significativas (Figura 1). A mediana da densidade óssea na coluna lombar foi de $0,923 \mathrm{~g} / \mathrm{cm}^{2}$ $(0,74-1,22)$ e no colo do fêmur foi de $0,88 \mathrm{~g} / \mathrm{cm}^{2}$ (0,51-1,61). Na Tabela 1 estão apresentados os dados relativos a todas as pacientes.

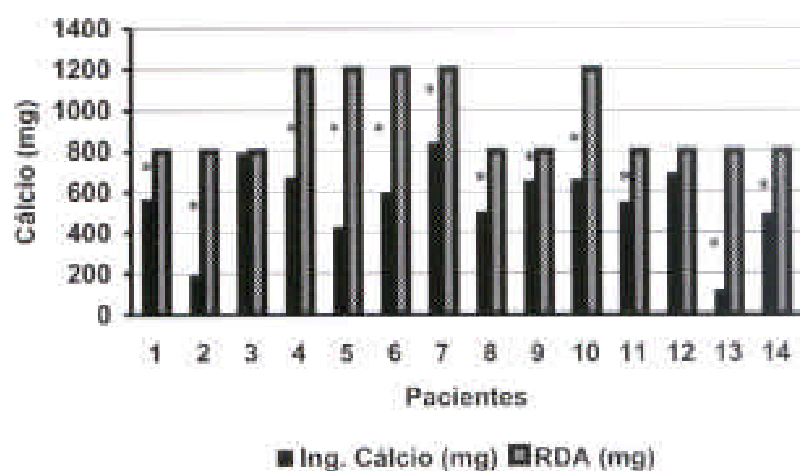

Figura 1 - Comparação entre a ingestão diária de cálcio calculada a partir do registro alimentar e o recomendado segundo o RDA (Recommended Dietary Allowances, 1989) ${ }^{6}$ para 0 cálcio (ingestão de cálcio recomendada pelo RDA: $800 \mathrm{mg} / \mathrm{dia}$ para mulheres entre10 e 18 anos e $1200 \mathrm{mg} / \mathrm{dia}$ para mulheres entre 19 e 50 anos). ${ }^{*} p<0,05$.

Houve correlação significativa entre o tempo de amenorréia e a perda de massa óssea, tanto na coluna lombar $(r=-0,65 ; p=0,01)$, como no fêmur $(r=-0,71 ; p=0,0068)$ (Figura 2A e B).

Houve uma relação entre o déficit de ingestão de cálcio e a perda de massa óssea. Das seis pacientes que apresentavam déficit na ingestão de cálcio menor que $60 \%$, a avaliação da DMO da coluna lombar e do colo do fêmur revelou osteopenia em quatro e três pacientes, respectivamente, e nenhuma apresentava osteoporose. Das oito pacientes que apresentavam déficit na ingestão diária de cálcio maior que 60\%, a DMO revelou que duas tinham osteopenia na coluna 
lombar e uma no colo do fêmur, e três apresentavam osteoporose na coluna lombar e três no colo do fêmur (Tabela 1).

Embora tenha havido uma relação, não houve uma correlação significativa entre a deficiên- cia na ingestão de cálcio e a perda de massa óssea na coluna lombar $(r=-0,35 ; p=0,26)$ e no colo do fêmur ( $r=-0,38 ; p=0,20)$, calculado com base nos desvios-padrão abaixo dos valores da normalidade (Figuras 3A e B).
A

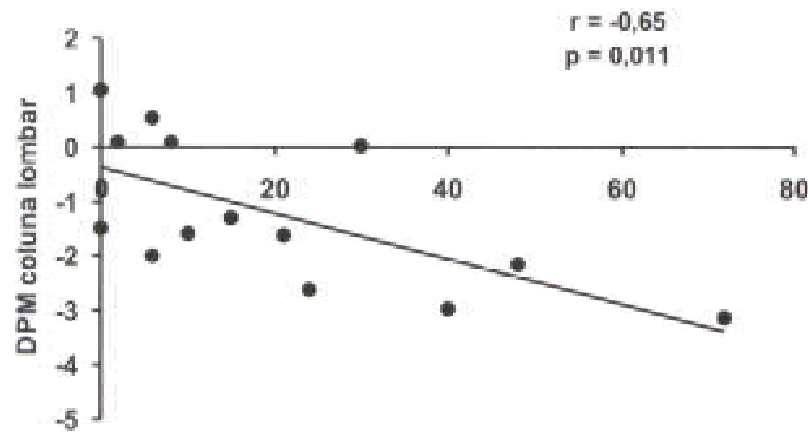

Tempo de amenorrèia (meses)

Figura 2 - Correlação entre desvio padrão da média (DPM) das densidades ósseas medidas na coluna lombar e no colo do fêmur e o tempo de amenorréia das pacientes com anorexia nervosa.

A - Coluna lombar

$B$ - Colo do fêmur
B

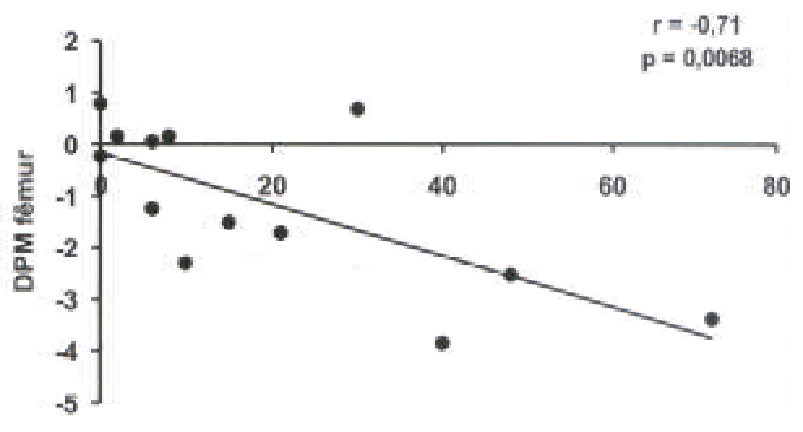

Tempo de amenorréia (meses)

Tabela 1 - Dados relativos à ingestão (déficits de ingestão) diária de cálcio e densidade óssea na coluna lombar e colo do fêmur em mulheres com anorexia nervosa.

\begin{tabular}{|c|c|c|c|c|}
\hline Paciente & $\begin{array}{l}\text { Ingestão de } \\
\text { cálcio (mg/dia) }\end{array}$ & $\begin{array}{l}\text { Déficit na ingestão } \\
\text { de cálcio (mg/dia) }\end{array}$ & $\begin{array}{l}\text { Densidade óssea na } \\
\text { coluna lombar }\left(\mathrm{g} / \mathrm{cm}^{2}\right)\end{array}$ & $\begin{array}{l}\text { Densidade óssea no } \\
\text { colo do fêmur }\left(\mathrm{g} / \mathrm{cm}^{2}\right)\end{array}$ \\
\hline 1 & 568 & $-228,6$ & 0,75 & 0,519 \\
\hline 2 & 192 & $-604,6$ & 0,87 & 0,79 \\
\hline 3 & 792 & $-8,5$ & 0,74 & 0,569 \\
\hline 4 & 672 & $-523,4$ & 0,92 & 1,61 \\
\hline 5 & 432 & $-9,7$ & 1,14 & 0,994 \\
\hline 6 & 600 & $-594,8$ & 1 & 0,941 \\
\hline 7 & 840 & 358,4 & 0,91 & 0,806 \\
\hline 8 & 504 & 445,6 & 0,79 & 1 \\
\hline 9 & 656 & $-147,1$ & 0,85 & 0,656 \\
\hline 10 & 660 & $-538,4$ & 1,04 & 0,862 \\
\hline 11 & 552 & $-250,1$ & 1,22 & 1,003 \\
\hline 12 & 688 & $-163,4$ & 1,17 & 0,928 \\
\hline 13 & 120 & $-1015,3$ & 0,93 & 0,889 \\
\hline 14 & 488 & -471 & 0,96 & 0,949 \\
\hline Média & 554,5 & $-382,7$ & 0,95 & 0,885 \\
\hline Mediana & 584 & -402 & 0,92 & 0,89 \\
\hline Desvio padrão & 202,9 & 273,8 & 0,15 & 0,26 \\
\hline Mínimo & 120 & $-8,5$ & 0,74 & 0,52 \\
\hline Máximo & 840 & $-1015,3$ & 1,22 & 1,61 \\
\hline
\end{tabular}


A

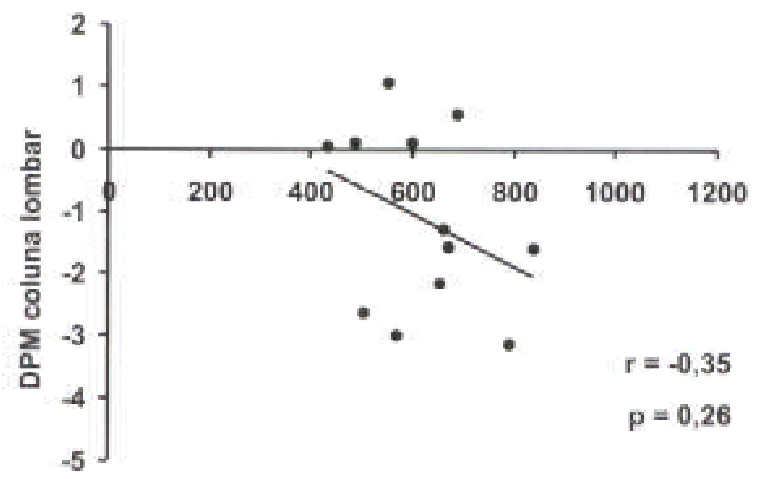

B

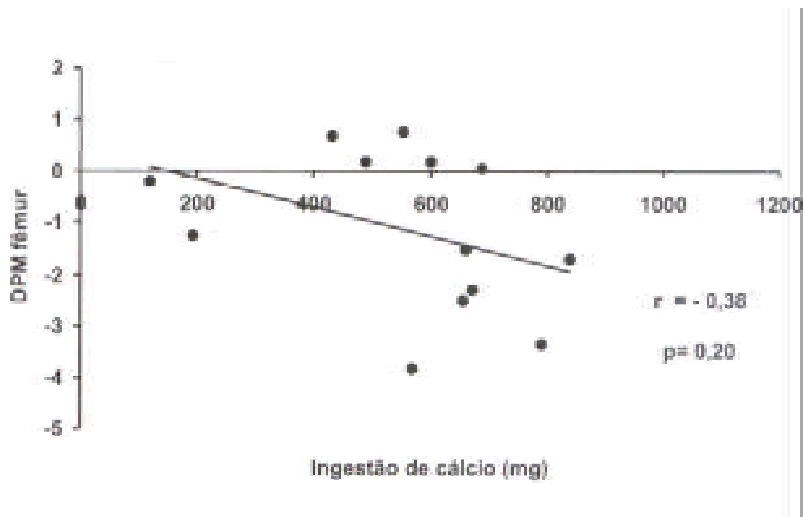

Figura 3 - Correlação entre desvio-padrão da média (DPM) das densidades ósseas medidas na coluna lombar e no colo do fêmur das pacientes com anorexia nervosa e a ingestão de cálcio.

A - Coluna lombar

B - Colo do fêmur

\section{Discussão}

As pacientes anoréxicas, por sua inadequada ingestão de alimentos, em geral apresentam quadro de desnutrição protéico-calórica em diferentes graus. Na avaliação do IMC as pacientes apresentam medianas abaixo do valor mínimo da faixa de normalidade, sendo este achado associado a quadros carenciais graves de vitaminas e minerais, entre os quais o cálcio ${ }^{7,8}$. Portanto, uma das conseqüências nutricionais mais importantes na evolução da AN é a carência de cálcio. Em condições de baixa ingestão prolongada de cálcio, esse passa a ser retirado de estruturas que o contêm, como os ossos, podendo provocar prejuízos de graus variáveis na sua densidade mineral.

Em comparação ao recomendado pelo RDA ${ }^{6}$ nossos resultados foram significativamente mais baixos. Ao se comparar a inadequação da ingestão de cálcio com a densidade óssea avaliada pela DMO verificamos que, embora a correlação não tenha sido significativa, há uma associação negativa entre o déficit na ingestão de cálcio e a massa óssea, ou seja, quanto maior o déficit de ingestão, maior tende a ser o desvio da normalidade. A ausência de significância poderia ser atribuída à casuística, que é relativamente pequena, ou à influência de outros fatores, como o tempo de doença e a quantidade de massa óssea adquirida até a instalação da AN.

Também se deve levar em conta a possibilidade de erro na coleta de informações da paciente sobre a sua alimentação, que é a base para o cálculo da ingestão de cálcio. Esta possibilidade é reduzida, porém possível, embora possa se tratar, em geral, de pacientes com razoável índice educacional. Vale lembrar que a AN é doença psiquiátrica envolvida por uma esfera de distúrbios dessa ordem, caracterizada pelo desejo de perder peso, medo mórbido de engordar e distorção da auto-imagem. Assim, não seria demais pensar que a informação da paciente anoréxica possa não refletir a realidade, estando a paciente se alimentando menos do que relata ao nutricionista.

Entretanto, quando comparamos a ingestão de cálcio com os valores das DMO, obtivemos resultados significativos. Verificou-se que no conjunto, praticamente dois terços das pacientes já apresentavam osteopenia ou osteoporose, seja no fêmur ou na coluna lombar. Considerando apenas aquelas nas quais o déficit de ingestão de cálcio foi inferior a $60 \%$ do mínimo desejável, a osteoporose não ocorreu em nenhuma paciente, ao passo que no grupo em que o déficit de ingestão foi maior que $60 \%$ do mínimo, quase $40 \%$ tinha osteoporose. Ou seja, há uma relação entre o déficit de ingestão de cálcio e a quantidade de massa óssea perdida.

As mulheres com AN apresentam, em determinado estágio do desenvolvimento da doença, distúrbios menstruais, caracterizados principalmente pela amenorréia. Em trabalho anterior mostramos que entre 64 pacientes anoréxicas avaliadas, nove não haviam apresentado menarca à época do seu primeiro atendimento, quarenta estavam amenorréicas e quinze tinham ciclos menstruais ${ }^{9}$. A amenorréia das mulheres com AN é do tipo central, por inibição da liberação pulsátil do GnRH hipotalâmico; como resultado as anoréxicas apresentam amenorréia com baixos níveis de gonadotrofinas e hipoestrogenismo.

Assim, poder-se-ia dizer que há relação entre o tempo de amenorréia e a instalação do hipoestrogenismo. Nesta linha de raciocínio, considerando-se o papel dos estrogênios no esta- 
belecimento da massa óssea nas mulheres, tem se sugerido que a osteoporose seja decorrente do hipoestrogenismo e, portanto, correlacionada ao tempo de amenorréia. De fato, em nossos dados encontramos forte correlação entre a densidade mineral óssea e o tempo de amenorréia, ou seja, quanto maior o tempo de amenorréia, maior o desvio da normalidade encontrado na massa óssea destas pacientes, seja na coluna como no fêmur.

Entretanto, a relação entre a perda de massa óssea e o hipoestrogenismo parece não ser tão direta assim, pois a simples reposição estrogênica, associada ou não a progestagênios, por si só não parece levar à recuperação da massa óssea ${ }^{10-12}$. Também a reposição de cálcio e complexos vitamínicos são inadequados para prevenir a perda de massa óssea. A utilização de agentes anabolizantes, como o rhIGF-1, associados a agentes anti-reabsortivos, como os contraceptivos orais, pode ser mais efetiva ${ }^{4}$. Estas associações estão ainda em fase de experimentação clínica, e as implicações e efeitos colaterais, a longo prazo, ainda não são bem conhecidos.

Concluindo, a maioria das pacientes com AN tem baixa ingestão de cálcio e perda significativa de massa óssea, que estão diretamente relacionadas entre si. A baixa densidade óssea também está correlacionada ao tempo de amenorréia. Desta forma a correção da ingestão de cálcio e o tratamento da amenorréia devem ser priorizados na abordagem destas pacientes para recuperação e/ ou prevenção de perda da massa óssea, mesmo levando-se em conta não serem estes os únicos fatores relacionados à osteopenia ou à osteoporose encontradas nestas mulheres.

\section{ABSTRACT}

Purpose: to determine daily calcium ingestion and its correlation with bone density in patients with anorexia nervosa.

Patients and Methods: fourteen women with anorexia nervosa recorded their $24 \mathrm{~h}$ food ingestion in a standardized diary and were submitted to bone densitometry. Data were analyzed statistically by the $\chi^{2}$ test and the Pearson correlation, with the level of significance set at $p<0.05$.

Results: mean bone density in the lumbar spine and in the femur were $0.95 \pm 0.15$ and $0.88 \pm 0.26$, respectively. There was a significant correlation between time of amenorrhea and bone mass loss both in the lumbar spine ( $r=-0.65$; $p=0.01)$ and in the femur $(r=-0.71 ; p=0.006)$. All patients but one presented lower calcium ingestion than the recommended dietary allowances, with a mean of $554.5 \mathrm{mg} /$ day (range from 120 to $840 \mathrm{mg} /$ day). Sixty-four percent of the patients presented some degree of bone mass loss (osteopenia or osteoporosis) in the spine and 57\% in the femur. None of the six patients with a deficit in calcium ingestion of less than $60 \%$ presented osteoporosis; on the other hand, three of eight patients who had deficit in calcium ingestion higher than $60 \%$ presented osteoporosis.

Conclusion: patients with anorexia nervosa had a low calcium ingestion and a significant bone mass loss, which were directly intercorrelated. Low bone density was also correlated with time of amenorrhea.

KEYWORDS: Anorexia nervosa. Bone mass. Osteoporosis.

\section{Referências}

1. Grinspoon S, Thomas E, Pitts S, et al. Prevalence and predictive factors for regional osteopenia in women with anorexia nervosa. Ann Intern Med 2000; 133:790-4.

2. Csermely T, Halvax L, Schmidt E, et al. A. Occurrence of osteopenia among adolescent girls with oligo/ amenorrhea. Gynecol Endocrinol 2002; 16:99-105.

3. Jagielska G, Wolanczyk T, Komender J, TomaszewiczLibudzic C, Przedlacki J, Ostrowski K. Bone mineral density in adolescents girls with anorexia nervosa - a cross sectional study. Eur Child Adolesc Psychiatry 2002; 11: 57-62.

4. Grinspoon S, Thomas L, Miller K, Herzog D, Klibanski A. Effects of recombinant human IGF-I and oral contraceptive administration on bone density in anorexia nervosa. J Clin Endocrinol Metab 2002; 87:2883-91.

5. American Psychiatric Association. Diagnostic and statistical manual of mental disorders. $4^{\text {th }}$ ed. Washington, DC: APA; 1994.

6. National Research Council, National Academy of Sciences, Food and Nutrition Board. Recommended dietary allowances. $10^{\text {th }}$ ed. Washington, DC: National Academy Press; 1989.

7. Muñoz MT, Morandé G, Garcia-Centenera JA, Hervás F, Pozo J, Argente J. The effects of estrogen administration on bone mineral density in adolescents with anorexia nervosa. Eur J Endocrinol 2002; 146:45-50.

8. Castro J, Toro J, Lazaro L, Pons F, Halperin I. Bone mineral density in male adolescents with anorexia nervosa. J Am Child Adolesc Psychiatry 2002; 41:613-8.

9. Ribeiro RPP, Santos PCM, Santos JE. Distúrbios de conduta alimentar: anorexia nervosa e bulimia nervosa. Medicina (Ribeirão Preto) 1998; 31:45-53.

10.Muñoz MT, Argente J. Anorexia nervosa: hypogonadotrophic hypogonadism and bone mineral density. Horm Res 2002; 57 (Suppl 2):57-62.

11. Muñoz MT, Argente J. Anorexia nervosa in female adolescents: endocrine and bone mineral density disturbances. Eur J Endocrinol 2002; 147:275-86.

12.Klibanski A, Biller BM, Schoenfeld DA, Herzog DB, Saxe VC. The effects of estrogen administration on trabecular bone loss in young women with anorexia nervosa. J Clin Endocrinol Metab 1995; 80:898-904.

Recebido em: 1/12/2003 Aceito com modificações em: 7/1/04 